\title{
Expression of mammalian target of rapamycin in atherosclerotic plaques is decreased under diabetic conditions: A mechanism for rapamycin resistance
}

\author{
YAN ZHANG* , QIANG WANG*, DACHUN YANG, DE LI, BING TANG, YONGJIAN YANG and SHUANGTAO MA \\ Department of Cardiology, General Hospital of PLA Chengdu Military Area Command, Chengdu, Sichuan 610083, P.R. China
}

Received September 11, 2013; Accepted March 13, 2014

DOI: $10.3892 / \mathrm{mmr} .2014 .2141$

\begin{abstract}
Our previous study demonstrated that diabetes increases in-stent restenosis following rapamycin-eluting stent placement, which was defined as rapamycin resistance. However, the underlying mechanisms of rapamycin resistance remain to be determined. In the present study, male apolipoprotein E-deficient $\left(\mathrm{ApoE}^{-/-}\right)$mice were randomly divided into control and diabetic groups. Diabetes was induced by injecting streptozocin (STZ). The hyperglycemic state, defined as a fasting plasma glucose level $>13 \mathrm{mmol} / \mathrm{l}$, was maintained for 8 weeks. At the end of the administration, the plasma levels of triglycerides (TG) and total cholesterol (TC) were significantly elevated in the diabetic group compared with the control mice (all $\mathrm{P}<0.01$ ). The present study revealed that diabetes increased the atherosclerotic plaque size of the aortic root $(\mathrm{P}<0.01)$ and the content of vascular smooth muscle cells (VSMCs) in the atherosclerotic lesion $(\mathrm{P}<0.01)$. Furthermore, the protein expression and phosphorylation of mammalian target of rapamycin (mTOR), 4E-binding protein 1 and ribosomal S6 kinase $1 \quad(\mathrm{P}<0.01)$ were significantly decreased in the diabetic mice compared with the control group. The decrease in the expression and phosphorylation of mTOR and its downstream kinases may be one of the molecular mechanisms underlying rapamycin resistance.
\end{abstract}

\section{Introduction}

Arteriosclerotic coronary disease is now the leading cause of mortality worldwide (1). Percutaneous transluminal coronary

Correspondence to: Dr Yongjian Yang or Dr Shuangtao Ma, Department of Cardiology, General Hospital of PLA Chengdu Military Area Command, 270 Tianhui Road, Rongdu Avenue, Chengdu, Sichuan 610083, P.R. China

E-mail: yangyongjian38@sina.com

E-mail: shuangtaoma@yahoo.com

*Contributed equally

Key words: diabetes, rapamycin, mammalian target of rapamycin, in-stent restenosis angioplasty has become the most common strategy for the treatment of severe coronary artery disease. Restenosis is a potential limitation of percutaneous coronary angioplasty. Although intracoronary stents reduce the incidence of restenosis following percutaneous coronary intervention (PCI), in-stent stenosis remains an important issue (2). Restenosis following PCI is predominantly caused by the proliferation of vascular smooth muscle cells (VSMCs) (3). The introduction of drug eluting stents (DES) has significantly decreased the rates of restenosis by inhibiting the accumulation and proliferation of VSMCs. Nevertheless, in-stent restenosis following PCI remains a challenging clinical problem despite the use of DES (4). It has been reported that the rate of restenosis remains at 7-16\% in diabetic patients who have received DES (5). Our previous study demonstrated that diabetes increases restenosis following rapamycin-eluting stent placement (6), indicating that diabetes remains an important predictor for restenosis in the DES era. When rapamycin becomes less effective at preventing in-stent restenosis, this condition is termed 'rapamycin resistance' (6). However, the underlying mechanisms of rapamycin resistance remain to be determined.

The mammalian target of rapamycin (mTOR) is a serine/threonine kinase that is important in mediating the proliferation of VSMCs (7). The best characterized downstream targets of mTOR are 4E-binding protein 1 (4EBP1) and ribosomal S6 kinase 1 (S6K1) (8). The activation of mTOR leads to the phosphorylation of 4EBP1 and S6K1, leading to the promotion of the initiation and elongation phases of mRNA translation and consequently the stimulation of VSMC proliferation. Rapamycin is a specific inhibitor of mTOR and suppresses the proliferation of VSMCs through inhibiting the mTOR-mediated proliferative signaling pathway (9). Therefore, the expressional and functional status of mTOR may affect the antiproliferative effect of rapamycin.

The present study hypothesized that diabetes may affect the expression of mTOR and its downstream kinases, attenuate the action of rapamycin and cause the clinical phenomena of rapamycin resistance. As a proof-of-concept study, the present study assessed how diabetes affected the growth of atherosclerotic lesions and mTOR expression in atherosclerotic plaques of apolipoprotein E-deficient $\left(\mathrm{ApoE}^{-/}\right)$mice. 


\section{Materials and methods}

Animal care. Experimental procedures were approved by the Hospital Animal Care and Use Committee (General Hospital of PLA Chengdu Military Area Command (Chengdu, China). In total, 16 male $\mathrm{ApoE}^{-/-}$mice (age, 6-8 weeks) were purchased from Beijing Yi-Ke-Li-Hao Biotechnology Co., Ltd. (Beijing, China). Animals were housed under a $12 \mathrm{~h} / 12 \mathrm{~h}$ day/night cycle with access to food and water ad libitum. The mice were fed a normal animal diet.

Animal model. The mice were randomly divided into two groups: The control group $(n=8)$ and the diabetes group $(n=8)$. The mice in the diabetic group were intraperitoneally injected with $55 \mathrm{mg} / \mathrm{kg}$ streptozocin (STZ; in $0.05 \mathrm{~mol} / \mathrm{l}$ citrate buffer; pH 4.5; Sigma, St. Louis, MO, USA) for five consecutive days. The mice in the control group were injected with an equal volume of citrate buffer. Fasting tail-blood glucose concentration (FBG) level was measured using a glucose test strip (Roche Diagnostics GmbH, Mannheim, Germany). Animals were considered to be diabetic if their FBG level was $>13 \mathrm{mmol} / \mathrm{l}$. The FBG levels of all mice were monitored at baseline and at weeks 1, 3 and 8 after injection of STZ.

Serum lipids. Fasting blood samples were obtained at the end of the experiments. The triglycerides (TG), total cholesterol (TC), low-density lipoprotein cholesterol (LDL-C) and high-density lipoprotein cholesterol (HDL-C) levels were measured by colorimetric assays using a commercially available kit (Jiancheng Bioengineering Institute, Nanjing, China) according to our previous study (10).

Atherosclerosis analysis. Atherosclerotic lesions in the aortic roots were examined in cross sections of the aortic origin according to a previous study (11). The sections $(5 \mu \mathrm{m})$ were stained with hematoxylin and eosin, and images were captured on an Olympus BX41 microscope (Olympus Corp., Tokyo, Japan). The atherosclerotic lesion area was quantified using Nikon NIS-Elements Research software (Nikon, Tokyo, Japan).

Immunohistochemistry. The protein expression of smooth muscle (SM) $\alpha$-actin and mTOR in atherosclerotic plaques was detected by immunohistochemical staining (12). Aortic roots were fixed in $4 \%$ paraformaldehyde for $12 \mathrm{~h}$, embedded in paraffin and then cut into sections $(5 \mu \mathrm{m})$. The sections were incubated with anti-SM $\alpha$-actin (BM002) and mTOR (Ab-2448) antibodies (diluted 1:50). Specific binding was detected using complexes of biotinylated goat anti-rabbit IgG secondary antibody and horseradish peroxidase using ABC kits (Boster Bioengineering Co., Wuhan, China). The antigen-antibody complex was subsequently visualized with DAB solution (Boster Bioengineering Co.). The sections were viewed under a light microscope. For the detection of positive staining, the portion of the color spectrum representing the positive signal for each stain was defined using the color cube-based method in Image-Pro plus 6.0 (Media Cybernetics, Inc., Bethesda, MD, USA). This color definition was then applied uniformly to all digitized images for that stain to determine the total positive area within the plaque and this positive area measurement was then normalized to the total area of the plaque. Additionally, the mean light density of the positive area was calculated.

Western blot analysis. Protein lysates were obtained by homogenizing aortic tissues with lysis buffer containing $1 \%$ Triton X-100, $150 \mathrm{mmol} / \mathrm{l} \mathrm{NaCl}, 1 \mathrm{mmol} / \mathrm{l}$ EDTA, $2.5 \mathrm{mmol} / \mathrm{l}$ sodium pyrophosphate, $1 \mathrm{mmol} / 1 \beta$-glycerophosphate, $1 \mathrm{mmol} / 1 \mathrm{Na}_{3} \mathrm{VO}_{4}, 1 \mu \mathrm{g} / \mathrm{ml}$ leupeptin, $1 \mu \mathrm{g} / \mathrm{ml}$ aprotinin and $20 \mathrm{mmol} / \mathrm{l}$ Tris ( $\mathrm{pH}$ 7.5) (13). The protein concentration was determined using Bio-Rad protein assay reagent (Bio-Rad, Hercules, CA, USA). Equal quantities of protein from the heart extracts were separated by SDS-PAGE (12\%). The samples were then electroblotted onto a nitrocellulose membrane (Boehringer Mannheim Corporation, Indianapolis, IN, USA) and probed with antibodies against mTOR (Ab-2448, rabbit polyclonal), phospho-mTOR-Ser2448 (p-mTOR; Ab-11221, rabbit polyclonal), phospho-4EBP1-Ser 65/Thr 70 (p-4EBP1; sc-12884, goat polyclonal), phospho-S6K1 (p-S6K1; eptomics-1175-1, rabbit monoclonal) and glyceraldehyde 3 phosphate dehydrogenase (GAPDH; sc-365062, mouse monoclonal) overnight. Following washing, the membrane was incubated with a horseradish peroxidase-conjugated secondary antibody (1:1,000 dilution; Santa Cruz Biotechnology, Inc., Santa Cruz, CA, USA) and bound antibody was visualized using a colored reaction. The relative band densities were quantified by densitometry using the Multi-Analyst software package (Bio-Rad). The equal loading of protein was confirmed by measuring GAPDH expression.

Statistical analysis. Continuous data are presented as the mean \pm standard error of the mean. Comparisons between the two groups were determined by the independent samples t-test (SPSS, Inc., Chicago, IL, USA). P $<0.05$ was considered to indicate a statistically significant difference.

\section{Results}

Characteristics of diabetic ApoE ${ }^{-/-}$mice. The body weight of control $\mathrm{ApoE}^{-/-}$mice significantly increased 8 weeks after the start of the experiment. However, the body weight of diabetic $\mathrm{ApoE}^{-/-}$mice decreased marginally following STZ administration and was significantly lower than that of the control mice at the end of the experiment $(\mathrm{P}<0.01$; Fig. 1A). No significant changes in the fasting blood glucose level in the control mice were observed. However, the fasting glucose concentration of diabetic mice increased to a level $>20 \mathrm{mmol} / \mathrm{l} 3$ weeks after STZ injection and maintained the hyperglycemic state until the end of the experiment (Fig. 1B). The plasma TG, TC, LDL-C and HDL-C concentrations in the diabetic mice were significantly higher than those of the control mice (Fig. 1C; all $\mathrm{P}<0.01)$.

Diabetes enhances atherosclerotic lesions. At week 8 after STZ administration, the atherosclerotic plaque area of the aortic root was significantly increased in diabetic mice compared with the control group $(\mathrm{P}<0.01$; Fig. $2 \mathrm{~A}$ and $\mathrm{B})$.

Diabetes increases VSMC content in atherosclerotic plaques. The proliferation of VSMCs in the atherosclerotic plaque and 

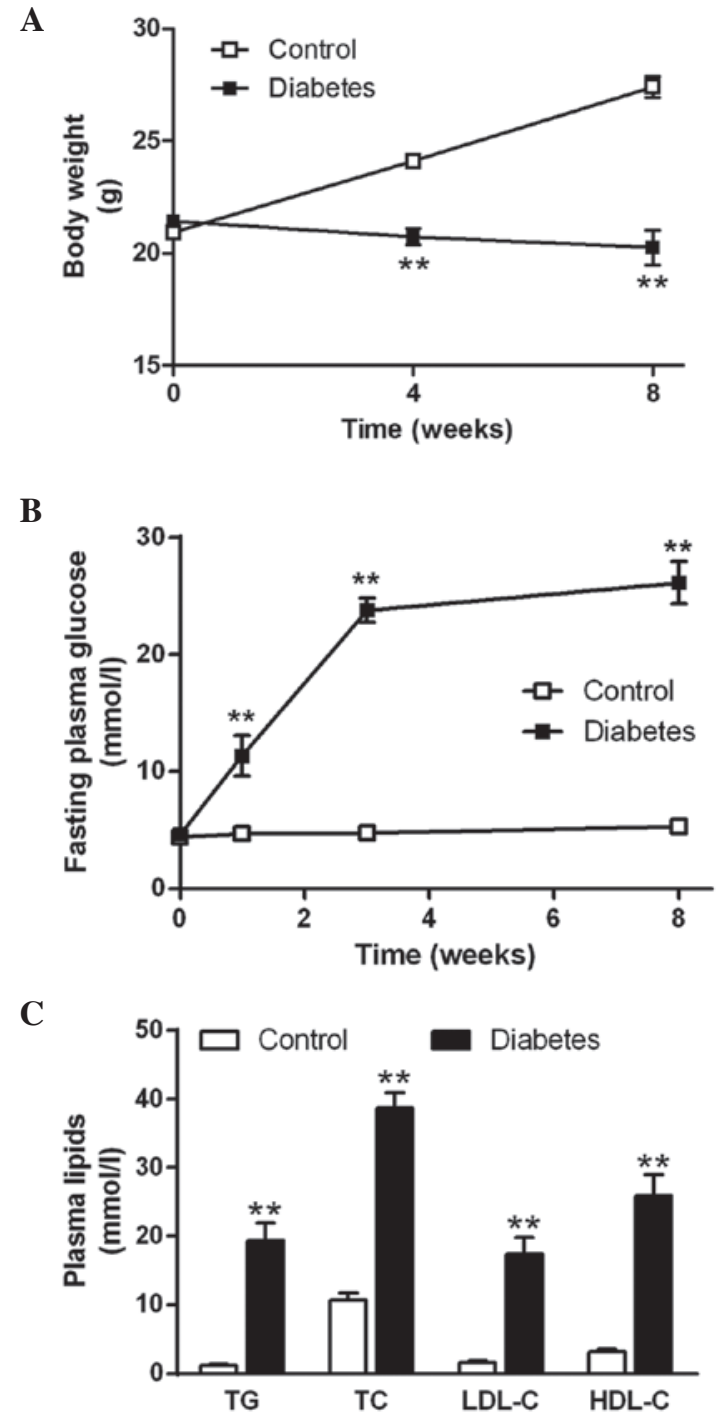

Figure 1. Characteristics of diabetic $\mathrm{ApoE}^{-/-}$mice. (A) Body weight of mice from the control and diabetic groups at baseline, week 4 and week 8 after STZ administration. (B) Fasting plasma glucose levels of mice from the control and diabetic groups at baseline, week 1, week 3 and week 8 after STZ administration. (C) TG, TC, LDL-C and HDL-C concentrations in $\mathrm{ApoE}^{-/}$mice in the control (open bars) and diabetic (solid bars) groups at week 8 after STZ treatment. The data are expressed as the mean \pm standard error of the mean; $n=8$ mice in the control group and $n=6$ in the diabetic group. ${ }^{* *} \mathrm{P}<0.01$ compared with the control group. STZ, streptozocin; TG, plasma triglyceride; TC, total cholesterol; LDL-C, low-density lipoprotein cholesterol; HDL-C, high-density lipoprotein cholesterol; $\mathrm{ApoE}^{-/}$, apolipoprotein E-deficient.

the vascular wall contributes to the development of restenosis. The VSMCs in atherosclerotic plaques were detected by immunohistochemistry staining. The content of VSMCs in diabetic mice expressed as the positive area or mean density was significantly higher than that in the control group $(\mathrm{P}<0.01$; Fig 3A, B and C).

Diabetes inhibits the mTOR signaling pathway. The mTOR signaling pathway and its downstream kinases have been viewed as the major regulators of the proliferation of VSMCs. The protein expression of mTOR in atherosclerotic plaques from diabetic mice was significantly decreased compared
A

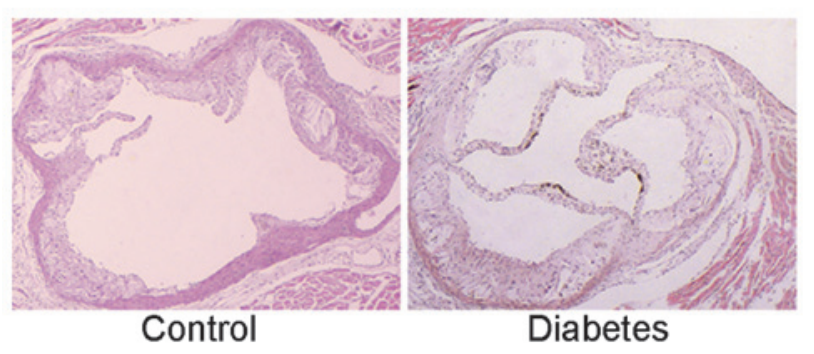

B

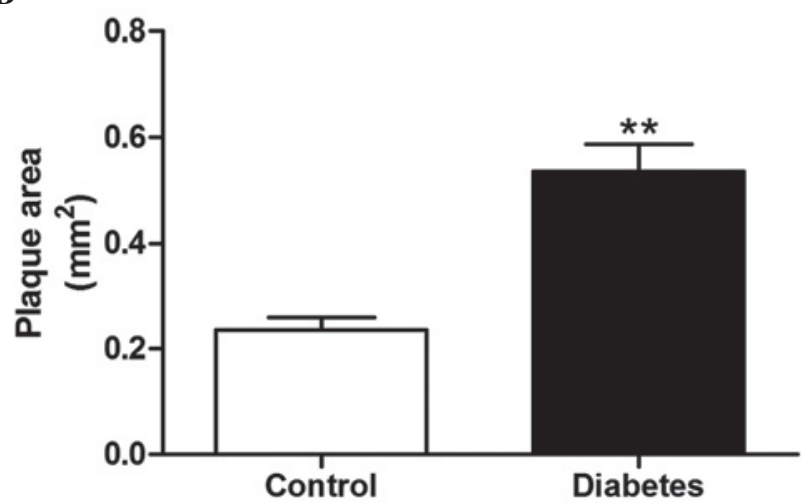

Figure 2. Diabetes enhances atherosclerotic lesions. (A) Representative images of the aortic root atherosclerotic lesions in $\mathrm{ApoE}^{-/}$mice from the control and diabetic groups stained with hematoxylin and eosin (magnification, $\mathrm{x} 10$ ). (B) Plaque size of the aortic root is expressed as the percentage of the aortic valvular area. The data are expressed as the mean \pm standard error of the mean; $\mathrm{n}=8$ mice in the control group and six mice in the diabetic group. ${ }^{* *} \mathrm{P}<0.01$ compared with the control group. ApoE ${ }^{-/}$, apolipoprotein E-deficient.

with that of the control animals $(\mathrm{P}<0.05$; Fig. $4 \mathrm{~A}$ and $\mathrm{C})$. The protein expression of mTOR, $\mathrm{p}$-mTOR and its major downstream kinases p-4EBP1 and p-S6K1 in aortic tissue were significantly downregulated in the diabetic mice compared with the control group (all $\mathrm{P}<0.01$; Fig. 4B and D).

\section{Discussion}

Our previous clinical follow-up study demonstrated that diabetes remains an independent risk factor for restenosis following DES in coronary arteries (6). There is also evidence that the efficacy of DES in the diabetic state varied with the type of stent (everolimus eluting stents and sirolimus eluting stents $>$ paclitaxel eluting stents and zotarolimus eluting stents) (14). These results suggest that diabetic patients have a distinct expressional model of the target of eluted drug. Therefore, the present study hypothesized that rapamycin resistance in diabetes is attributed to alterations in the expression of the rapamycin target mTOR and its downstream signaling pathway. The present study provides compelling evidence supporting the concept that diabetes not only significantly enhances the growth of atherosclerotic plaques and the proliferation of VSMCs, but also downregulates the expression of mTOR, 4EBP1 and S6K1. Therefore, alterations in the expression of the mTOR signaling pathway may mediate the clinical phenomenon of rapamycin resistance.

Although the macrophage-derived foam cell is important in the development of atherosclerosis, the migration and 
A

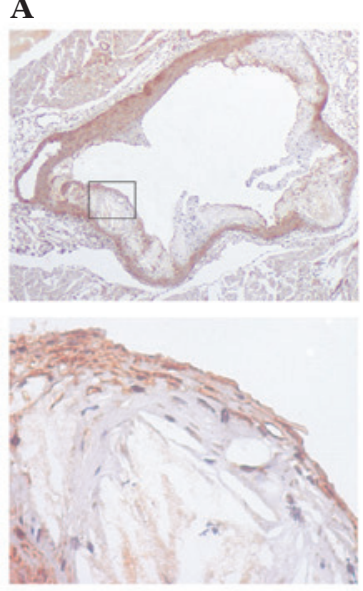

Control

$\mathbf{B}$

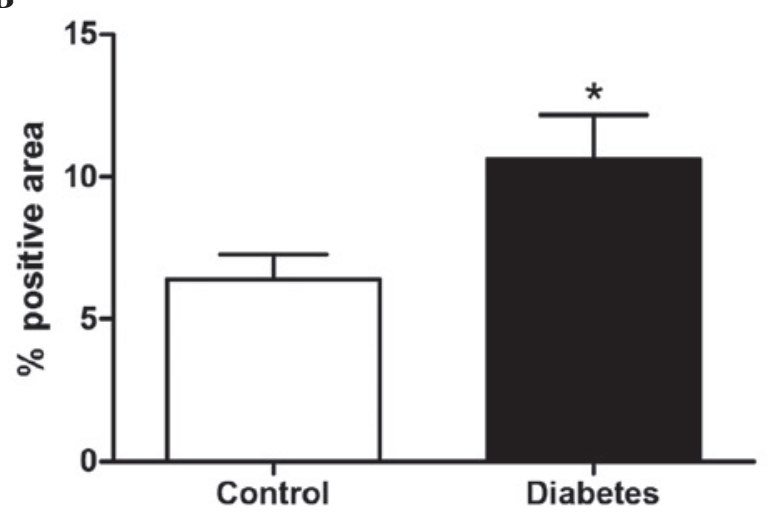

C

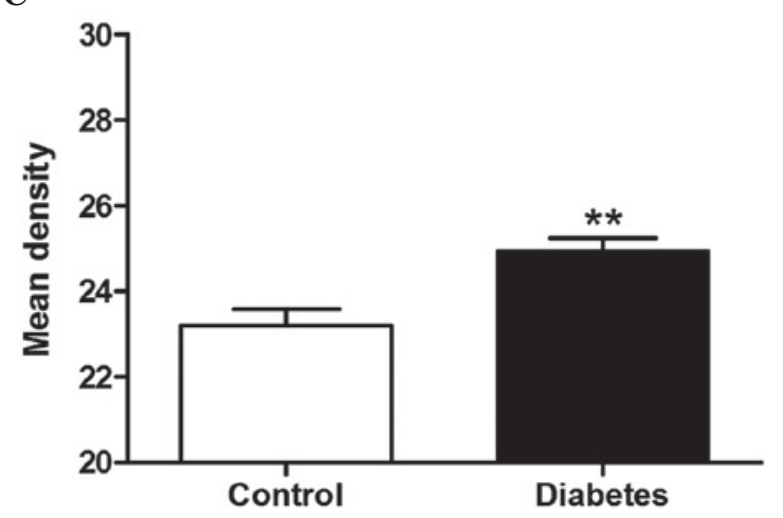

Figure 3. Diabetes increases VSMC content in the atherosclerotic plaque. (A) Representative immunohistochemistry staining images of VSMCs in aortic lesions from the control and diabetic mice. Upper panel, magnification, $\mathrm{x} 100$; lower panel, magnification, $\mathrm{x} 400$. (B and C) VSMC content is expressed as the area percentage and the mean density of the positively stained area (brown). The data are expressed as the mean \pm standard error of the mean; $\mathrm{n}=8$ mice in the control group and six mice in the diabetic group. ${ }^{*} \mathrm{P}<0.05$ and ${ }^{* *} \mathrm{P}<0.01$, compared with the control group. VSMC, vascular smooth muscle cell.

proliferation of VSMCs in the arterial intima are also key events in the formation of atherosclerotic lesions (15). The proliferation of VSMCs is not only involved in the formation of plaques, but also contributes to the process of in-stent restenosis. Growth factors, including platelet-derived growth factor, basic fibroblast growth factor and insulin-like growth factor-1, stimulate
A
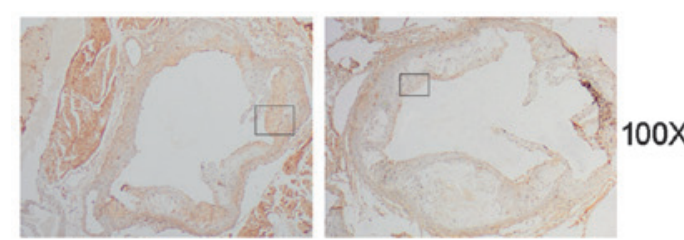

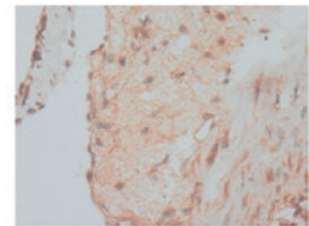

Control

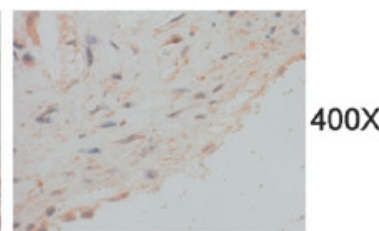

Diabetes
B

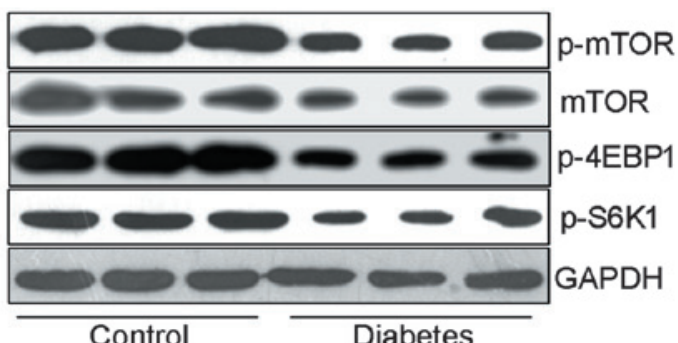

C

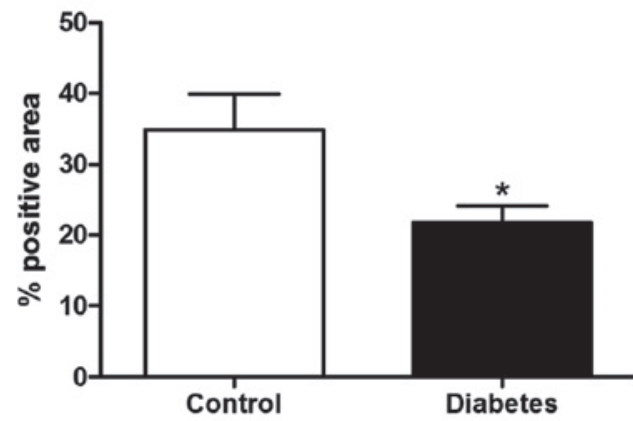

D

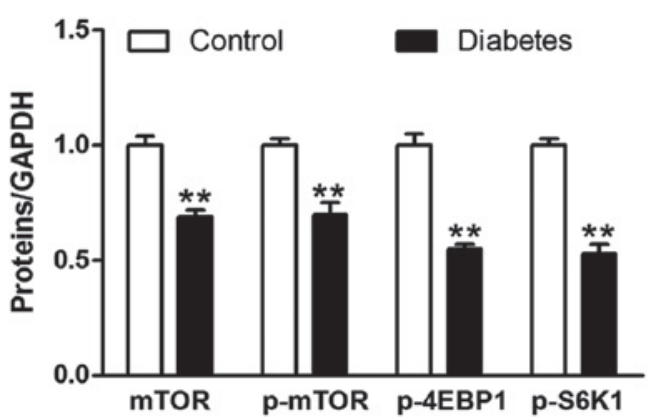

Figure 4. Diabetes blunts the mTOR signaling pathway. (A) Representative images of immunohistochemical staining demonstrating the expression of mTOR in the aortic atherosclerotic plaque. Upper panel, magnification, x100; lower panel, magnification, x400. (B) Protein expression of mTOR, p-mTOR, p-4EBP1 and p-S6K1 in aortic tissues was determined by western blotting using specific antibodies. Equal protein loading was confirmed using the GAPDH antibody. (C) Expression level of mTOR was determined by the positively stained area (brown) percentage. (D) Ratios of mTOR, p-mTOR, p-4EBP1 and p-S6K1 to GAPDH are shown in the bar graph. The data are expressed as the mean \pm standard error of the mean; $n=8$ mice in the control group and six mice in the diabetic group. ${ }^{*} \mathrm{P}<0.05$ and ${ }^{* *} \mathrm{P}<0.01$, compared with the control group. mTOR, mammalian target of rapamycin; p-mTOR, phosphor-mTOR; p-4EBP1, phospho-4E-binding protein 1; p-S6K1, phospho-ribosomal S6 kinase 1; GAPDH, glyceraldehyde-3-phosphate dehydrogenase. 
VSMCs to enter the cell cycle and proliferate (16). Currently, DESs delivering rapamycin, rapamycin analogues or paclitaxel were used to prevent in-stent restenosis through inhibiting the proliferation of VSMCs (17). The specific target of rapamycin is mTOR. The mTOR pathway regulates VSMC growth through effectors, including 4EBP1 and S6K1. Diabetic patients remain at an increased risk of developing in-stent restenosis despite the use of rapamycin eluting stents, which was observed in the present study and in other studies $(6,18)$. The phenomenon of rapamycin resistance may result from two distinct issues. One is that the mTOR signaling pathway is impaired in diabetes and the other is that the mTOR signaling pathway is over-activated by diabetes. A previous in vitro experiment demonstrated that increased leptin levels, which mimicked hyperleptinemia in diabetes and metabolic syndrome, may activate the mTOR signaling pathway in primary cultured VSMCs (19), suggesting that a higher dose of rapamycin may be more effective in limiting in-stent restenosis in the setting of hyperleptinemia. However, the present in vivo study demonstrated that the mTOR signaling pathway is downregulated in the setting of hyperglycemia. This result suggests that a higher dose of rapamycin is not an effective therapeutic strategy to limit in-stent restenosis.

Based on the current available evidence, the critical mechanism mediating in-stent restenosis in the setting of diabetes has not been elucidated. As the mTOR signaling pathway has been demonstrated to be inhibited by diabetes, certain other signaling pathways may exhibit compensatory upregulation or activation. The underlying mechanisms controlling the proliferation of VSMCs in the setting of hyperglycemia remain to be revealed in future studies. The in-stent restenosis in diabetes may be resolved by using two or multiple DES.

In conclusion, decreases in the expression and phosphorylation of mTOR and its downstream kinases may be one of the molecular mechanisms underlying rapamycin resistance. Future investigation is required to identify potential targets involved in in-stent restenosis under diabetic conditions.

\section{Acknowledgements}

This study was supported by grants from the National Natural Science Foundation of China (grant nos. 81070191 and 81100232).

\section{References}

1. Ohira T and Iso H: Cardiovascular disease epidemiology in Asia: an overview. Circ J 77: 1646-1652, 2013.
2. Indermuehle A, Bahl R, Lansky AJ, et al: Drug-eluting balloon angioplasty for in-stent restenosis: a systematic review and meta-analysis of randomised controlled trials. Heart 99: 327-333, 2013.

3. Chaabane C, Otsuka F, Virmani R and Bochaton-Piallat ML: Biological responses in stented arteries. Cardiovasc Res 99: 353-363, 2013.

4. De Caterina AR, Cuculi F and Banning AP: Incidence, predictors and management of left main coronary artery stent restenosis: a comprehensive review in the era of drug-eluting stents. EuroIntervention 8: 1326-1334, 2013.

5. Pate GE, Lee M, Humphries K, et al: Characterizing the spectrum of in-stent restenosis: implications for contemporary treatment. Can J Cardiol 22: 1223-1229, 2006.

6. Ma S, Yang D, Zhang X, et al: Comparison of restenosis rate with sirolimus-eluting stent in STEMI patients with and without diabetes at 6-month angiographic follow-up. Acta Cardiol 66: 603-606, 2011.

7. Trovati M, Doronzo G, Barale C, Vaccheris C, Russo I and Cavalot F: Leptin and vascular smooth muscle cells. Curr Pharm Des: May 14, 2013 (Epub ahead of print).

8. Chong ZZ and Maiese K: Mammalian target of rapamycin signaling in diabetic cardiovascular disease. Cardiovasc Diabetol 11: 45, 2012.

9. Li W, Li Q, Qin L, et al: Rapamycin inhibits smooth muscle cell proliferation and obstructive arteriopathy attributable to elastin deficiency. Arterioscler Thromb Vasc Biol 33: 1028-1035, 2013.

10. Ma S, Yang D, Li D, Tang B and Yang Y: Oleic acid induces smooth muscle foam cell formation and enhances atherosclerotic lesion development via CD36. Lipids Health Dis 10: 53, 2011.

11. Jin F, Jiang S, Yang D, et al: Acipimox attenuates atherosclerosis and enhances plaque stability in ApoE-deficient mice fed a palmitate-rich diet. Biochem Biophys Res Commun 428: 86-92, 2012.

12. Jiang S, Jin F, Li D, et al: Intermittent hypobaric hypoxia promotes atherosclerotic plaque instability in ApoE-deficient mice. High Alt Med Biol 14: 175-180, 2013.

13. Ma S, Yang D, Li D, Tang B, Sun M and Yang Y: Cardiac extracellular matrix tenascin-C deposition during fibronectin degradation. Biochem Biophys Res Commun 409: 321-327, 2011.

14. Bangalore S, Kumar S, Fusaro M, et al: Outcomes with various drug eluting or bare metal stents in patients with diabetes mellitus: mixed treatment comparison analysis of 22,844 patient years of follow-up from randomised trials. BMJ 345: e5170, 2012.

15. Koga $\mathrm{J}$ and Aikawa M: Crosstalk between macrophages and smooth muscle cells in atherosclerotic vascular diseases. Vascul Pharmacol 57: 24-28, 2012.

16. Jung F, Haendeler J, Goebel C, Zeiher AM and Dimmeler S: Growth factor-induced phosphoinositide 3-OH kinase/Akt phosphorylation in smooth muscle cells: induction of cell proliferation and inhibition of cell death. Cardiovasc Res 48: $148-157,2000$.

17. Marx SO, Totary-Jain H and Marks AR: Vascular smooth muscle cell proliferation in restenosis. Circ Cardiovasc Interv 4: 104-111, 2011.

18. Scheen AJ, Warzée F and Legrand VM: Drug-eluting stents: meta-analysis in diabetic patients. Eur Heart J 25: 2167-2168, 2004.

19. Shan J, Nguyen TB, Totary-Jain H, Dansky H, Marx SO and Marks AR: Leptin-enhanced neointimal hyperplasia is reduced by mTOR and PI3K inhibitors. Proc Natl Acad Sci USA 105: 19006-19011, 2008. 